\title{
The Madurai Hypoglycaemia Survey: a snapshot of hypoglycaemic (un)awareness in India
}

\author{
KRISHNAN SWAMINATHAN
}

\begin{abstract}
Aim: To assess prescribing practices and patient awareness of issues relating to hypoglycaemia in a South Indian population.

Methods: A standard questionnaire was administered to one hundred consecutive patients with diabetes attending the Diabetes Clinic at Apollo Specialty Hospital, Madurai, India. All data were tabulated and analysed using Microsoft Excel 2007.

Results: There was a predominance of sulphonylurea use, in combination with oral agents and insulin. Nearly $60 \%$ of patients reported that their doctor never talked about hypoglycaemia during clinic visits. At least $20 \%$ of patients had at least one hospital admission related to a hypoglycaemic event since the diagnosis of diabetes. More than a third of patients were not aware of symptoms of hypoglycaemia nor did they know how to manage the symptoms.

Conclusions: Medical professionals in India need to do more to educate patients about issues relating to hypoglycaemia, its importance, awareness and appropriate management.

Br J Diabetes Vasc Dis 2015;15:17-19
\end{abstract}

Key words: hypoglycaemia, education, diabetes mellitus, autonomic neuropathy, self management

\section{Introduction}

Hypoglycaemia remains an important factor limiting good glycaemic control. Severe hypoglycaemia (hypoglycaemia requiring third party assistance) is one of the most feared acute complications in diabetes. Apart from significantly affecting QoL, ${ }^{1}$ the concern with hypoglycaemia is the potential link with vascular events and death. ${ }^{2-5}$ Acute hypoglycaemia evokes profound physiological changes that protect the brain from neuroglycopaenia. However, some of these changes may have an adverse

Address for correspondence: Dr Krishnan Swaminathan Consultant Endocrinologist, Kovai Medical Center \& Hospital, Coimbatore, India 625020

Tel: 00918526421150

E-mail: drkrishnanswaminathan@kmchhospitals.com

http://dx.doi.org/10.15277/bjdvd.2014.037

\author{
Abbreviations and acronyms \\ EHS Edinburgh Hypoglycaemia Scale \\ OBSTACLE An observational Study to Assess Correlation between \\ Glycemic Control and Hypoglycemia in Patients \\ with Type 2 Diabetes Treated with Sulfonylureas \\ QoL Quality of life
}

effect on the vasculature that is already damaged. Plausible mechanisms by which hypoglycaemia can cause or aggravate cardiovascular disease include sympatho-adrenal activation, increased thrombogenesis, abnormal cardiac depolarisation, vasoconstriction and inflammation. ${ }^{6}$ Until recently, there has always been an uncertainty about the direct causal relationship between hypoglycaemia, death and vascular outcomes.2,7 However, in a large metaanalysis of 903,510 subjects with type 2 diabetes, a bias analysis indicated that the observed association between severe hypoglycaemia and cardiovascular disease may not be entirely due to confounding by severe comorbid illness. ${ }^{8}$ This recent study gives additional support to the notion that avoiding hypoglycaemia, especially severe hypoglycaemia, may be important in preventing cardiovascular disease. We therefore set out to assess prescribing practices and patient awareness of issues relating to hypoglycaemia in the south Indian city of Madurai.

\section{Patients and methods}

One hundred consecutive patients with known type 1 or type 2 diabetes of more than one year's duration attending the outpatient department at Apollo Specialty Hospital, Madurai for the first time were included in this study. Exclusion criteria were patients who were already under the care of the study consultant, patients on diet alone and patients with less than one year's duration of diabetes. Type 2 diabetes was defined as any patient with diabetes diagnosed at $>30$ years of age, initiated on oral agents. Type 1 diabetes was defined as any patient with diabetes $<30$ years of age, initiated on insulin the onset of diabetes. Local Ethics Committee approval was obtained prior to the study. A standard questionnaire (Figure 1) was administered to all patients by the same doctor evaluating the patient's awareness of symptoms of hypoglycaemia, cut-off values, response to hypoglycaemia, number of self-reported hypoglycaemia episodes over the preceding three months, number of admissions related to hypoglycaemia and whether the patient's doctor had discussed hypoglycaemia. Additional data on age, duration, type of 
Figure 1. Hypoglycaemia questionnaire used in the study

\begin{tabular}{l} 
HYPOGLYCAEMIA QUESTIONNAIRE: MADURAI HYPO SURVEY \\
Name: \\
Age: \\
Sex: \\
Duration of diabetes: \\
Type of diabetes: \\
Type of treatment: \\
What are the symptoms of hypoglycaemia? \\
(Edinburgh Hypoglycaemia Scale (EHS): Sweating, confusion, shaking, \\
hunger, confusion, drowsiness, odd behaviour, speech difficulty, \\
incoordination, headache and nausea) \\
1=Correct answer (at least 5 of EHS) \\
2=Wrong answer or Don't know \\
3=Partially correct (1-4 of EHS) \\
What is the cut-off value for hypoglycaemia? \\
1=Correct answer (<70mgs/dl) \\
2=Wrong answer (anything <60 or $>70)$ or Don't know \\
3=Acceptable answer (between $60-70)$ \\
What do you do when you have a hypo? \\
1=Correct answer (15-20 gms carbs, cup of juice, one soda or \\
4 teaspoons of sugar, 3-4 small biscuits) \\
2=Wrong answer or Don't know \\
3=Acceptable (small variations of above + chocolates) \\
How many episodes of hypo symptoms have you had over the last \\
3 months? \\
How many hospitalisations with hypoglycaemia have you had since \\
diagnosis of diabetes? \\
Has your doctor talked about hypoglycaemia? \\
\hline
\end{tabular}

diabetes and treatment were also collected. All results were recorded and analysed using Microsoft Excel 2007.

\section{Results}

A total of one hundred patients had the hypoglycaemia questionnaire administered (Figure 1). The results are shown in Table 1. The mean age of patients was 53.3 years with a male predominance (54 males and 46 females). The mean duration of diabetes was 7.5 years. Ninety-five patients had type 2 diabetes and five had type 1 diabetes. Thirty-five percent of patients were on sulphonylurea therapy (with or without addition of other oral agents). 33\% were on non-sulphonylurea based regimes (metformin, gliptins, acarbose and pioglitazone alone or combined), $20 \%$ were on insulin (on its own or combined with metformin) and $12 \%$ were on a combination of insulin with a sulphonylurea. Nearly $40 \%$ of patients were not aware of the symptoms of hypoglycaemia or how to deal with a hypoglycaemic event. More than two-thirds of the patients were not aware of the cutoff value for hypoglycaemia. Nearly $50 \%$ of patients had 1-5
Table 1 Results from the hypoglycaemia questionnaire

\begin{tabular}{ll}
\hline Total number of patients ( $\mathrm{n})$ & 100 \\
\hline Male/ Female & $54 / 46$ \\
\hline Mean duration of diabetes (years) & 7.5 years \\
\hline Type 2/ Type 1 (n) & $95 / 5$ \\
\hline Sulphonylurea based regimes & $47 \%$ \\
$\begin{array}{l}\text { Self reported hypoglycaemia over } \\
\text { preceding } 3 \text { months (1-5 episodes) }\end{array}$ & $50 \%$ \\
\hline
\end{tabular}

self-reported hypoglycaemic symptoms over the preceding three months. One patient reported approximately five episodes of hypoglycaemia per week on a combination of insulin and sulphonylyurea with no change made to his medications. Nearly $20 \%$ of patients reported at least one hospitalisation with hypoglycaemia since the diagnosis of diabetes. Finally, about $60 \%$ of patients reported that their doctors had never mentioned issues relating to hypoglycaemia during their clinic visits.

\section{Discussion}

There is an adage often quoted in medicine, "All drugs are dangerous and how dangerous a drug depends on the skill of the prescriber". This is very true in diabetes management, as all the glucose lowering medications have their own unique side effects, with one of the main concerns being the risk of hypoglycaemia. There are two ways to reduce the risk of hypoglycaemia: one is for the healthcare professional to use glucose lowering medications of low hypoglycaemic potential and the other is to empower and educate the patient about symptoms of hypoglycaemia so that corrective steps can be taken rapidly. The frequency of hypoglycaemia is often underestimated in diabetes. For example, the UK Hypoglycaemia Study Group showed that the rates of severe hypoglycaemia in type 2 diabetes patients treated with insulin or sulphonlyureas of less than two years' duration was similar at 7\%. The prevalence of mildly symptomatic hypoglycaemia with sulphonylureas in this study was around $40 \% .^{9}$

There are specific factors in India that may greatly predispose patients to a higher risk of hypoglycaemia compared with western counterparts. The results from the recently published OBSTACLE Hypoglycaemia Study from India showed that glimeperide was the most commonly used sulphonylurea and there was a weakly positive but statistically significant correlation between its doses and hypoglycaemic scores. ${ }^{10}$ In this study, self reported hypoglycaemia was observed in $50 \%$ of patients initiated on sulphonylurea and the rates were highest during the initial weeks of sulphonlyurea therapy. In addition, we also observed continued sulphonylurea use in addition to insulin, which greatly increases the risk of hypoglycaemic events, especially in the elderly.

Other factors we observed as a generalisation include:

- poor patient education,

- $\quad$ perceived lack of time for health care professionals due to busy clinic schedules, 
- absence of a strong diabetes specialist nurse team,

- poor utilisation of home blood glucose monitoring in indicated patients,

- cultural factors,

- cost of medicines.

Most of the studies in Asia on the subject of hypoglycaemia are in the context of Ramadan, comparing sulphonylureas and gliptins. ${ }^{11,12}$ Our aim was therefore to do a real-world study on patient awareness about issues relating to hypoglycaemia. Our study confirms the predominant use of sulphonylureas, with nearly $50 \%$ of patients on sulphonylureas, either alone or in combination with other oral agents and insulin. About two-thirds of patients on insulin or sulphonylurea in our study reported at least 1-5 episodes of hypoglycaemia over the preceding three months, but this increased to $81 \%$ when insulin was combined with a sulphonylurea. There is evidence of more hypoglycaemia and weight gain when insulin is combined with sulphonylureas, compared with insulin plus metformin..$^{13}$ Therefore, counselling of patients is mandatory if the physician decides to continue secretagogues with insulin. Unfortunately, about $60 \%$ of patients in our study reported that their doctor had never spoken about hypoglycaemia during their visits. Not surprisingly, $80 \%$ of our patients were not aware of the cut-off values for hypoglycaemia and $40 \%$ of patients did not know the symptoms of hypoglycaemia or what to do at the time of a hypoglycaemic event. This lack of knowledge leads to unnecessary hospital admissions and emergency call-outs, escalating costs and anxiety for the patients and their families. There is good evidence for both apparent and hidden costs of hypoglycaemia in terms of medical resource utilisation, disturbance of daily life and time taken off work. 9,14 Nearly 20\% of the patients in our study had at least $1-5$ hospitalisations with hypoglycaemia.

Our study has its own limitations. The sample size was small for a questionnaire study. There was no attempt to minimise bias based on demographics or socio-economic status. Observer bias is possible as the questionnaire was administered by a doctor, which could have influenced the responses.

While complete avoidance of hypoglycaemia is impossible with any diabetes treatment, targeted education regarding hypoglycaemia to all our patients with diabetes will be of great benefit in reducing the morbidity associated with hypoglycaemia. Overall, our study gives a snapshot of the immense amount of work that healthcare professionals need to do in India to update and improve practice, educate and empower patients and train specialist nurses and diabetes educators to reduce the burden of hypoglycaemia in clinical practice.

\section{Conflict of interest None. \\ Funding None.}

Acknowledgements The author was formerly a Consultant at Apollo Speciality Hospital, Madurai and wishes to acknowledge the management and staff for their help with this study.

\section{References}

1. Rombopoulos G, Hatzikou M, Latsou D, et al. The prevalence of Hypoglycaemia and its impact on the quality of life (QoL) of type 2 diabetes

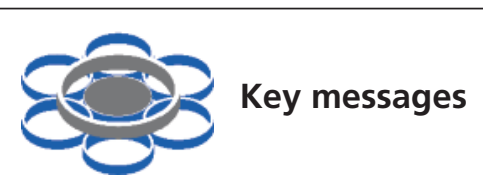

- Hypoglycaemia is a limiting factor for good glycaemic control

- Patient education will reduce morbidity associated with hypoglycaemia

- Diabetes Educators and Specialist Nurses are the need of the hour in the Indian subcontinent

mellitus patients (The HYPO Study). Hormones (Athens) 2013;12:5508. http://dx.doi.org/10.14310/horm.2002.1443

2. Zoungas S, Patel A, Chalmers J, et al; ADVANCE Collaborative Group. Severe Hypoglycaemia and risks of vascular events and death. $N$ Engl $J$ Med 2010;363:1410-8. http://dx.doi.org/10.1056/NEJMoa1003795

3. Kosiborod M, Inzucchi SE, Goyal A, et al. Relationship between spontaneous and iatrogenic hypoglycaemia and mortality in patients hospitalized with acute myocardial infarction. JAMA 2009;301:1556-64. http://dx.doi.org/10.1001/jama.2009.496

4. Sechterberger MK, Bosman RJ, Oudemans-van Straaten HM, et al. The effect of diabetes mellitus on the association between measures of glycaemic control and ICU mortality: a retrospective cohort study. Crit Care 2013;17:R52

5. Svensson AM, McGuire DK, Abrahamsson P, et al. Association between hyper- and hypoglycaemia and 2 year all-cause mortality risk in diabetic patients with acute coronary events. Eur Heart J 2005;26:1255-61. http://dx.doi.org/10.1093/eurheartj/ehi230

6. Wright RJ, Frier BM. Vascular disease and diabetes: is hypoglycaemia an aggravating factor? Diabetes Metab Res Rev 2008;24:353-63. http://dx.doi.org/10.1002/dmrr.865

7. Bonds DE, Miller ME, Bergenstal RM, et al. The association between symptomatic, severe hypoglycaemia and mortality in type 2 diabetes: retrospective epidemiological analysis of the ACCORD study. BMJ 2010; 340:b4909. http://dx.doi.org/10.1136/bmj.b4909

8. Goto A, Arah OA, Goto M, et al. Severe hypoglycaemia and cardiovascular disease: systematic review and meta-analysis with bias analysis. BMJ 2013;347:f4533. http://dx.doi.org/10.1136/bmj.f4533

9. Heller SR, Choudhary P, Davies C, et al. UK Hypoglycaemia Study Group. Risk of hypoglycaemia in types 1 and 2 diabetes: effects of treatment modalities and their duration. Diabetologia 2007:50:1140-7. http://dx.doi.org/10.1007/s00125-007-0599-y

10. Kalra S, Deepak MC, Narang $P$, et al. Usage pattern, glycaemic improvement, hypoglycaemia, and body mass index changes with sulfonylureas in real-life clinical practice: results from OBSTACLE Hypoglycaemia Study. Diabetes Technol Ther 2013;15:129-35. http://dx.doi.org/10.1089/dia.2012.0237

11. Al-Arouj M, Hassoun AA, Medlej R, et al. The effect of vildagliptin relative to sulphonylureas in Muslim patients with type 2 diabetes fasting during Ramadan: the VIRTUE study. Int J Clin Pract 2013;67:957-63.

12. Aravind SR, Ismail SB, Balamurugan $R$, et al. Hypoglycaemia in patients with type 2 diabetes from India and Malaysia treated with sitagliptin or a sulfonylurea during Ramadan: a randomized, pragmatic study. Curr Med Res Opin 2012;2:1289-96. http://dx.doi.org/10.1185/03007995.2012.707119

13. Swinnen SG, Dain MP, Mauricio D, et al. Continuation versus discontinuation of insulin secretagogues when initiating insulin in type 2 diabetes. Diabetes Obes Metab 2010:12:923-5. http://dx.doi.org/10.1111/j.1463-1326.2010.01258.x

14. Willis WD, Diago-Cabezudo JI, Madec-Hily A, et al. Medical resource use, disturbance of daily life and burden of hypoglycaemia in insulintreated patients with diabetes: results from a European online survey. Expert Rev Pharmacoecon Outcomes Res 2013;13:123-30 http://dx.doi.org/10.1586/erp.12.80 